\title{
COMPOSITION AND DIVERSITY IN SOIL SAMPLES FROM THE NAPAHAI PLATEAU WETLAND OF SOUTHWESTERN CHINA
}

\author{
CHEN, W. ${ }^{1}-\mathrm{LI}, \mathrm{X} . \mathrm{R}^{2}{ }^{2}-\mathrm{TENG}, \mathrm{P} . \mathrm{Y}^{1}-\mathrm{WEI}, \mathrm{Y} . \mathrm{L}^{2}{ }^{2}-\mathrm{JI}, \mathrm{X} . \mathrm{L}^{{ }^{2 *}}$ \\ ${ }^{I}$ Medical School, Kunming University of Science and Technology, Kunming, Yunnan Province \\ 650500, China
}

${ }^{2}$ Faculty of Life Science and Technology, Kunming University of Science and Technology, Kunming, Yunnan Province 650500, China

*Corresponding author

e-mail: jixiuling@126.com

(Received $30^{\text {th }}$ May 2019; accepted $3^{\text {rd }}$ Sep 2019)

\begin{abstract}
The Napahai plateau wetland, located in northwestern Yunnan province, China, is a unique seasonal plateau wetland of low-latitude and high-altitude, yet the microbial community is still unknown. To address this shortcoming, MiSeq high-throughput sequencing was used to analyze the composition and diversity of bacteria and archaea. The bacterial community comprised of 64 phyla, 164 classes and 484 genera, in which Proteobacteria was the most abundant, followed by Actinobacteria, Chloroflexi and Acidobacteria. Archaea comprised of 3 phyla, 5 classes, and 7 genera, in which Thaumarchaeota dominated. The results indicated that the composition and diversity of the bacterial community were more influenced by seasonal changes than by soil types, whereas the archaeal community was mostly resistant to these factors. Additionally, canonical correlation analysis (CCA) showed that the diversity of the bacterial community was closely correlated with nitrogen $(\mathrm{N})$, total nitrogen $(\mathrm{TN})$ and soil organic matter (SOM) in the dry season. However, no significant correlation of any of the factors was observed in the archaeal community. In conclusion, these results indicated that microbial communities in the soil of Napahai plateau wetland have unique diversity and composition, and Napahai plateau wetland as a microbial resource requires protection and restoration.
\end{abstract}

Keywords: wetland ecosystem, high throughput sequencing, bacterial community diversity, archaeal community diversity, environmental factors

\section{Introduction}

Wetlands are important links between terrestrial and aquatic systems. They are large reservoirs of biodiversity and ecologically powerful natural ecosystems. Ecological services of wetlands have high social and economic value. In wetland ecosystems, microbial community diversity is much better than vegetation species diversity to reflect environmental changes. The microbial community diversity and composition of wetland soils are more sensitive and comprehensive to reflect the wetland ecological conditions. Also they have a great significance for maintaining the balance of wetland ecosystems, repairing damaged wetlands and carrying out comprehensive environmental management.

Recently, the rapid development of high-throughput DNA sequencing has allowed the detailed study of the diversity of microbial communities in wetland soils, with fruitful results. For instance, soil samples were collected from six wetlands on the QinghaiTibetan plateau, and high-throughput 16S rRNA gene sequencing was used to assess the composition and localization of enriched microbial communities. Overall, microbial communities from the Qinghai-Tibetan plateau wetlands showed significant potential in converting cellulose and chitin to methane at low temperatures (Dai et al., 2016). In a subarctic wetland in Russia, the major bacterial groups identified in peat by high- 
throughput sequencing of the 16S rRNA genes were Acidobacteria (35.4-41.2\%), Alphaproteobacteria (19.1-24.2\%), and Gammaproteobacteria (7.9-11.1\%). The distinctive feature of this community was a high proportion of two subdivisions of Acidobacteria, which are not characteristic for boreal sphagnum peat bogs (Danilova et al., 2016). In coastal mangrove wetlands, MiSeq high throughput sequencing was used to understand the microbial composition and diversity pattern. The five most abundant phyla within the bacterial and archaeal communities remained stable between two distinctive seasons, suggesting that the microbial community in the Mai Po wetland exhibits a mild seasonal dynamic (Zhou et al., 2017).

In a wetland ecosystem, geographic distance and environmental factors are two important drivers of the distribution of microorganisms (Widder et al., 2014; Savio et al., 2015). However, in the whole space, environmental factors are more important. Previous studies have demonstrated that soil microbial communities can be significantly influenced by mineral nutrients (Su et al., 2015). In Qinghai-Tibet plateau, soil organic matter and $\mathrm{pH}$ were the main factors affecting the soil macrofauna communities of composition and diversity. In addition to environmental factors, the sampling site can influence the microbial community. For instance, Cao studied soil samples across a regional scale in China (including Jiangxi, Hubei, and Henan provinces). Henan has the largest amount of bacterial and fungal phospholipid fatty acids (PLFAs), while Jiangxi shows the lowest amount of PLFAs (Cao et al., 2016). Moreover, the dominant microbial communities generally differ in different sites. For instance, the dominant bacterial phyla are Proteobacteria, Bacteroidetes, and Actinobacteria in Beijing (Tian et al., 2014), but they are Proteobacteria, Acidobacteria, and Chloroflexi in Jiangsu (Zhao et al., 2014).

The Napahai plateau wetland, an important water source for the upper reaches of the Yangtze River and located in northwestern of Yunnan Province, China, is a unique, seasonal plateau wetland in low latitude and at high altitude. The Napahai plateau wetland has been listed as an internationally important wetland, and has the significance of being a representative plateau wetland (Xiang et al., 2018). However, the microbial community composition and diversity of this region have not become the subjects of research. Soil microbes have ecological functions of central importance, playing crucial roles in nutrient cycling and soil fertility. Their diversity is a sensitive indicator of soil quality that can determine its ecological function (Anderson et al., 2009). Thus, research on microbial communities in the Napahai plateau wetland has become a fundamental need. To provide new insight into the Napahai plateau wetland microbial resources, six sets of samples from three different soil types were studied, with high-throughput 16S rRNA gene sequencing used to investigate the bacterial and archaeal community diversity in the soil. The major aims of this research were to address the following two key questions. (i) What are the characteristics of the bacterial and archaeal communities, with respect to diversity in the soil of Napahai plateau wetland? (ii) What are the influential factors and their contributions to the compositions of the two communities within the Napahai wetland environments?

\section{Materials and methods}

\section{Soil sample sites and collection}

Soil samples were collected from the Napahai plateau wetland, where the whole year split between the dry season (October to April) and the rainy season (May to September). According to the region's geomorphology, samples were collected from the areas of three 
different soil types, including bog soil (YN), swamp meadow soil (SD) and peat soil (NT).

Soil samples were collected in November 2014 (dry season) and June 2015 (rainy season) from three sampling areas (YN, SD and NT). For each sampling areas, two samples were collected and for each sample, and eight mixed sample plot soils were selected ("S" distribution) at $10 \mathrm{~cm}$ soil layer. Table 1 shows the general conditions of each sample, and Figure 1 shows the sampling sites. YN samples were collected from the vicinity of Lalang from areas less disturbed by human activities. The work regarded as the environmental background area. NT soil sample area was overgrazed by livestock, belonging to a tourism hotspot. Stellera chamaejasme Linn., a plant that is a sign of wetland degradation, was also found. Therefore, this area was regarded to have significant disturbance by human activities. The soil from YN, SD and NT sampling areas represented the transition from native swamp soil and swamp meadow soil to meadow soil formed due to the degradation of the Napahai plateau wetland. The twelve soil samples were obtained and stored at $4{ }^{\circ} \mathrm{C}$, and then transported to the lab for DNA extraction and property analysis.

\section{Analysis of physical and chemical properties}

Soil samples for property analysis were crushed and sifted using sieves with apertures of $1 \mathrm{~mm}$. Physical and chemical factors, including soil organic matter (SOM), total nitrogen (TN), total phosphorus (TP), total potassium (TK) and nitrogen (N), were measured using ion chromatography (Dionex ${ }^{\mathrm{TM}}$ ICS-6000, Thermo Scientific, USA) at the Ministry of Agriculture Agricultural Products Quality Supervision and Testing Center (Kunming, China).

\section{DNA extraction and PCR amplification}

Total soil DNA was extracted from $0.1 \mathrm{~g}$ of each soil sample, which had roots removed and was ground using the Power Soil DNA kit (MoBio Laboratories Inc., CA, USA) according to the manufacturer's instructions (Zhou et al., 2017). The DNA was confirmed using $1.0 \%$ agarose gel electrophoresis and a NanoDrop (ND2000, Thermo Fisher, USA), then stored at $-80{ }^{\circ} \mathrm{C}$.

For bacterial and archaeal communities, the primer pairs bac-515F 5'GTGCCAGCMGCCGCGGTAA3', and bac-806R 5'GGACTACHVGGGTWTCTAAT3'; arc-U515F 5'CAGYMGCCRCGGKAAHACC3' and arc-U806R 5'GGACTACNSGGGTMTCTAAT3' were used for amplifying the V4 region of the $16 \mathrm{~S}$ rRNA genes of bacterial and archaeal, respectively, including barcodes and adapters (Peiffer et al., 2013; Shehab et al., 2013). The PCR mixtures (50 $\mu \mathrm{l}$ ) contained $25 \mu \mathrm{l} 2 \times$ MightyAmp Buffer Ver.2, $1 \mu \mathrm{l}$ of each primer $(10 \mu \mathrm{mol} / \mathrm{L}), 1.0 \mu \mathrm{l}$ MightyAmp DNA Polymerase, and 2.0 $\mu$ l template DNA (10-200 ng), with nuclease-free water added to $50 \mu \mathrm{l}$. The PCR amplifications used an initial denaturation step at $94{ }^{\circ} \mathrm{C}$ for $4 \mathrm{~min}$, followed by 30 cycles of $30 \mathrm{~s}$ at $94{ }^{\circ} \mathrm{C}, 45 \mathrm{~s}$ at $56^{\circ} \mathrm{C}$ and $35 \mathrm{~s}$ at $72{ }^{\circ} \mathrm{C}$ and were held at $72{ }^{\circ} \mathrm{C}$ for $10 \mathrm{~min}$. The $16 \mathrm{~S}$ rRNA gene PCR products were sequenced using the Illumina platform (Peiffer et al., 2013).

\section{Sequence analysis}

For each sample, three amplicons were pooled together and purified according to the Gene JET kit (Thermo Scientific, USA) instructions. Sequencing of the purified 
amplicons was performed on an Illumina MiSeq platform (MiSeq PE300, Illumina, USA) at the Computer Center (Beijing, China). Sequence analysis was performed with Fast Length Adjustment of Short reads (FLASH), discarding the low-quality sequences. Using UPARSE v7.1, sequences were clustered into operational taxonomic units (OTUs) with a 97\% similarity cutoff, and Usearch7.1 was used to filtered chimaeras (Edgar, 2013). The remaining sequences, including 530,418 bacterial and 561,217 archaeal, were assigned to OTUs at the 97\% similarity level using Mothur v1.34.4 (Schloss et al., 2009). Each representative OUT sequence was assigned to a taxonomic level using the Quantitative Insights into Microbial Ecology (QIIME) pipeline and the RDP classification method (Caporaso et al., 2010).

Table 1. The overview of Napahai plateau wetland sampling areas

\begin{tabular}{|c|c|c|c|c|}
\hline Soil samples & Sampling points & Longitude & Latitude & $\begin{array}{c}\text { Average altitude } \\
\text { (m) }\end{array}$ \\
\hline YN1 & NPH-YN1-YN8 & E99³7’43.00"-41.26” & 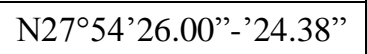 & 3290 \\
\hline YN2 & NPH-YN1'-YN8' & E99॰37’22.88”-20.75" & N2753'41.21”-36.81" & 3282 \\
\hline SD1 & NPH-SD1-SD8 & E99³8'6.46”-0.67” & N2751'36.33”-40.22" & 3277 \\
\hline SD2 & NPH-SD1'-SD8' & E99॰38'7.73"-15.63" & $\mathrm{N} 27^{\circ} 50 ’ 44.27 ”-36.02 "$ & 3275 \\
\hline NT1 & NPH-NT1-NT8 & E99³8’7.00”-3.63” & $\mathrm{N} 27^{\circ} 50^{\prime} 1.00 ”-49^{\prime} 58.92^{\prime \prime}$ & 3273 \\
\hline NT2 & NPH-NT1'-NT8', & E99³8’0.96”-1.97”' & N2749’35.63"-34.87” & 3272 \\
\hline
\end{tabular}

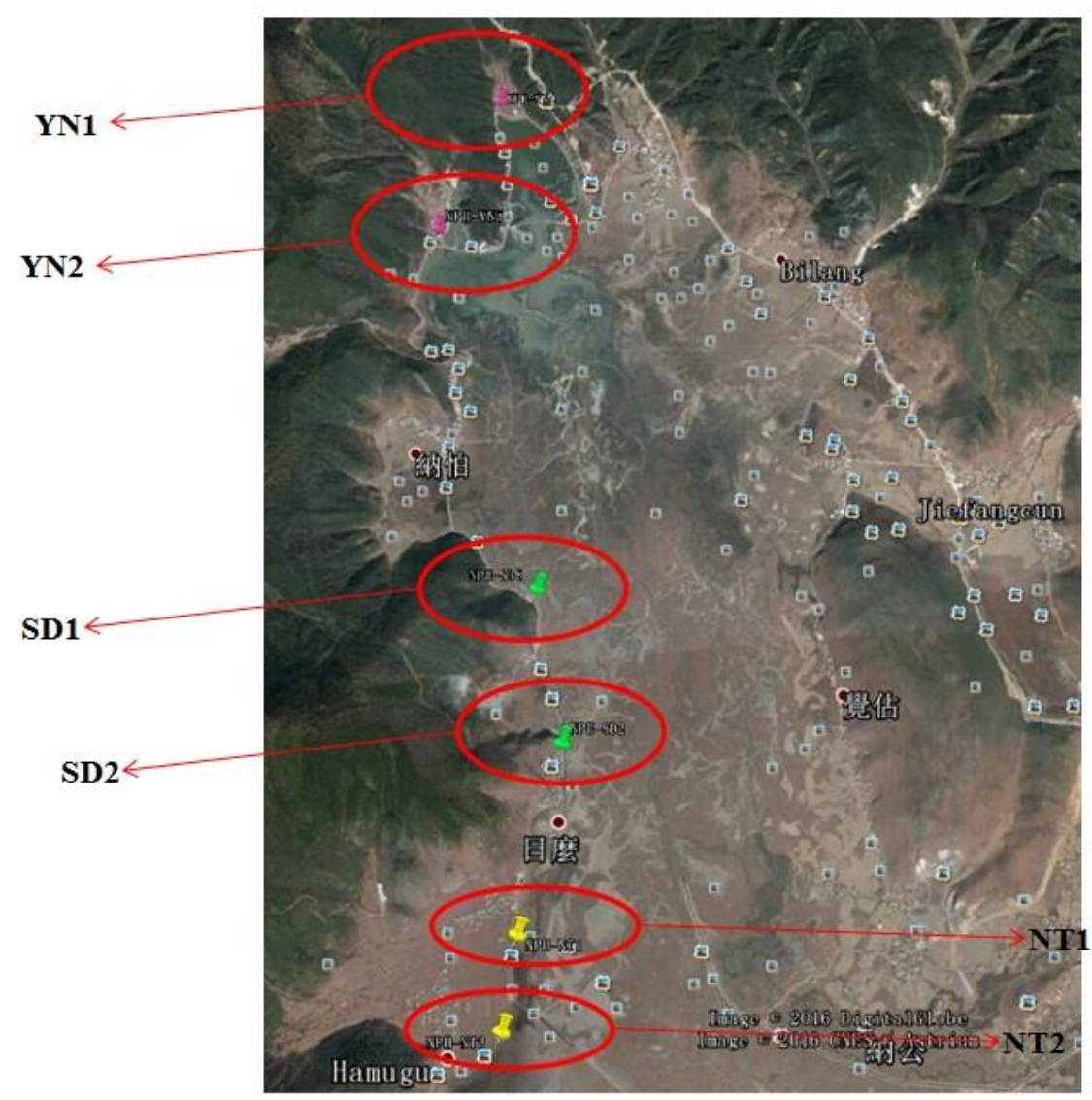

Figure 1. Distribution of Napahai plateau wetland sampling areas. Each sampling area is composed of eight sampling points and eight sampling points distributed as " $S$ " 


\section{Statistical analysis}

A number of alpha diversity indices were assessed at the $97 \%$ similarity level using mothur 1.34.4 including the Shannon-Wiener and Simpson index, the abundance based coverage estimator (ACE), terminal richness estimation (Chao1), and Good's coverage estimator (Schloss et al., 2009). Beta diversity was measured using Bray-Curtis distances among samples, and community differences were assessed using completelinkage clustering analysis. Based on Weighted_Unifrac, the Unweighted Pair-group Method with Arithmetic Means (UPGMA) was applied. The heatmap and Venn diagrams were generated using the Vegan Package for R v3.0.2 (Oksanen et al., 2013). Canonical correspondence analysis (CCA) was performed using Canoco v4.5.1 to reveal the relationships between microbial community diversity and soil environmental factors (Legendre et al., 2001).

\section{Results}

\section{Physical and chemical properties of soil samples}

Table 2 shows the physical and chemical properties of the soil. It can be concluded that soil nutrient contents ( $\mathrm{SOM}, \mathrm{TN}, \mathrm{TP}, \mathrm{TK}$ and $\mathrm{N}$ ) show no significant differences in different seasons $(p>0.05)$, but are significantly different in different soil types $(p<0.05)$.

Table 2. Determination of physical and chemical factors of soil samples

\begin{tabular}{c|c|c|c|c|c}
\hline Sample & SOM, g/kg & TN, $\mathbf{g} / \mathbf{k g}$ & $\mathbf{N}, \mathbf{~ m g} / \mathbf{k g}$ & $\mathbf{T P}, \mathbf{g} / \mathbf{k g}$ & $\mathbf{T K}, \mathbf{g} / \mathbf{k g}$ \\
\hline DS.YN1 & 105.8 & 0.568 & 393 & 0.032 & 0.416 \\
DS.YN2 & 117.4 & 0.624 & 368 & 0.062 & 0.414 \\
DS.SD1 & 31.2 & 0.26 & 121 & 0.102 & 0.664 \\
DS.SD2 & 26.8 & 0.166 & 97.9 & 0.106 & 0.675 \\
DS.NT1 & 43.7 & 0.445 & 246.3 & 0.279 & 0.795 \\
DS.NT2 & 38.7 & 0.392 & 233 & 0.369 & 0.806 \\
RS.YN1 & 145.8 & 0.659 & 381 & 0.088 & 0.647 \\
RS.YN2 & 160.9 & 0.823 & 346 & 0.081 & 0.643 \\
RS.SD1 & 22.3 & 0.18 & 106 & 0.09 & 0.732 \\
RS.SD2 & 17.4 & 0.156 & 91.3 & 0.127 & 0.764 \\
RS.NT1 & 59.7 & 0.435 & 259 & 0.12 & 0.950 \\
RS.NT2 & 51.2 & 0.358 & 227 & 0.142 & 0.908 \\
\hline
\end{tabular}

\section{Microbial community alpha-diversity analysis}

To estimate the bacterial and archaeal community diversity and richness, the work applied the Shannon-Wiener index and Simpson's index, ACE, Chao1 and Good's coverage estimation (Table 3). In general, high OTU richness in both the bacterial and archaeal communities was found. Good's coverage estimator showed high coverage and reasonable sequencing depth. Regarding the seasons, the diversity (Shannon-Wiener and Simpson's) and richness (ACE, Chao1) indices for the bacterial community showed higher diversity in the rainy season than in the dry season, whereas there was no obvious change of index in the archaeal community. The soil type also influenced the 
diversity of the bacterial and archaeal communities. In the NT sampling area, both the bacterial and archaeal communities had higher diversity than in the other sampling areas.

Table 3. The diversity analysis of bacterial and archaeal community at the $97 \%$ similarity level

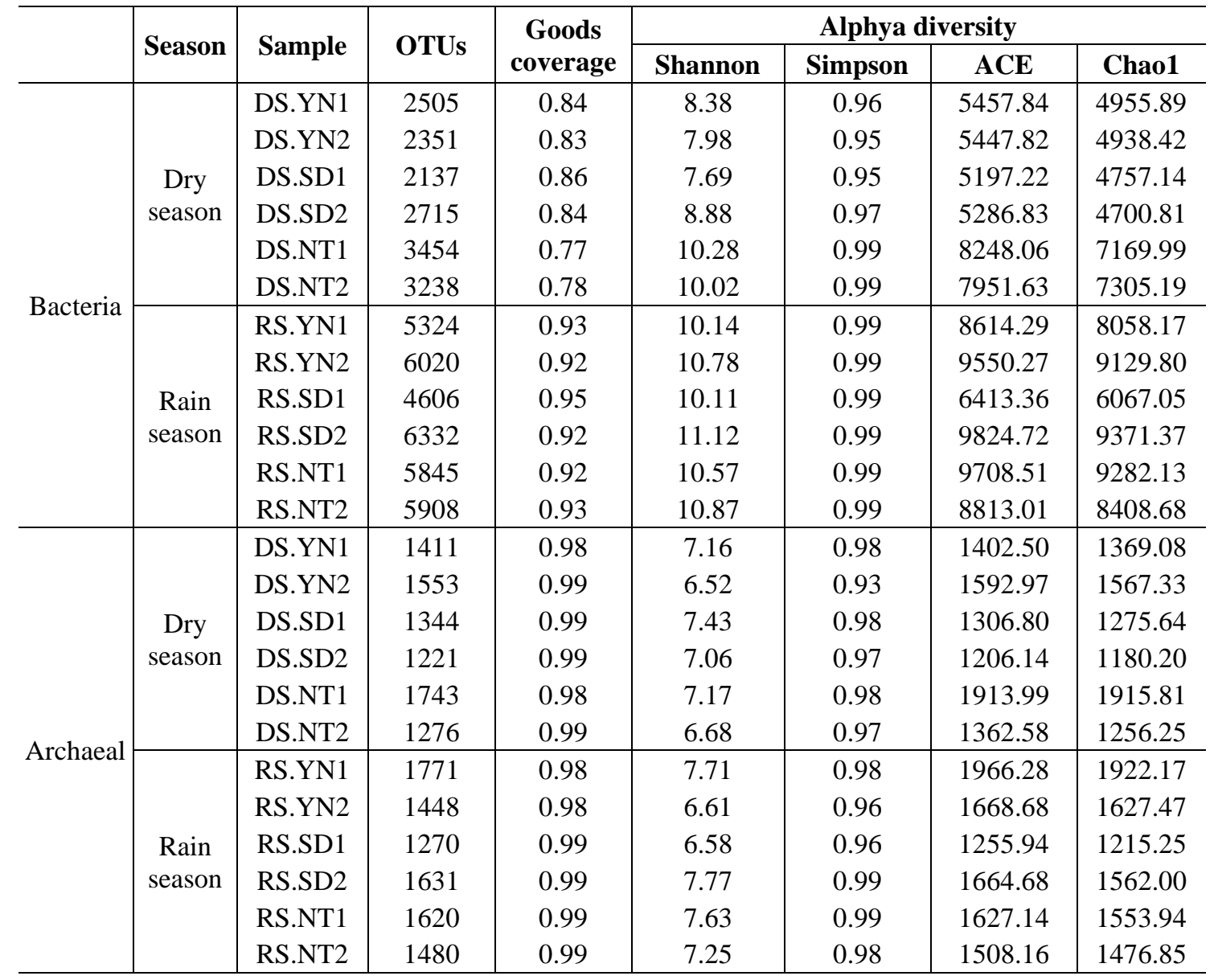

\section{Taxonomic composition of the microbial community}

Analysis of the bacterial community composition in the Napahai plateau revealed 64 phyla, 164 classes, 271 orders, 330 families and 484 genera. In addition, 3 archaeal phyla were detected to comprise 5 classes, 8 orders, 7 families and 7 genera. Figure 2 shows the relative abundances of bacteria and archaea at the phylum and genus levels.

The dominant bacterial phyla across all samples were Proteobacteria, Actinobacteria, Chloroflexi and Acidobacteria, accounting for 72.4 to $-81.8 \%$ of the bacterial sequences. It depended on the season and soil type (Fig. 3). The abundance of the phylum Proteobacteria was much higher $(p<0.1)$, and that of Chloroflexi and Acidobacteria was much lower $(p<0.1)$ in the dry season, while Proteobacteria, Chloroflexi and Acidobacteria showed the differences $(p<0.1)$ in soil type. The archaeal sequence information included approximately $40 \%$ unknown; the dominant archaeal phylum, Thaumarchaeota (Cenarchaeum), accounted for 50\% without exhibiting differences in seasons and soil types. 

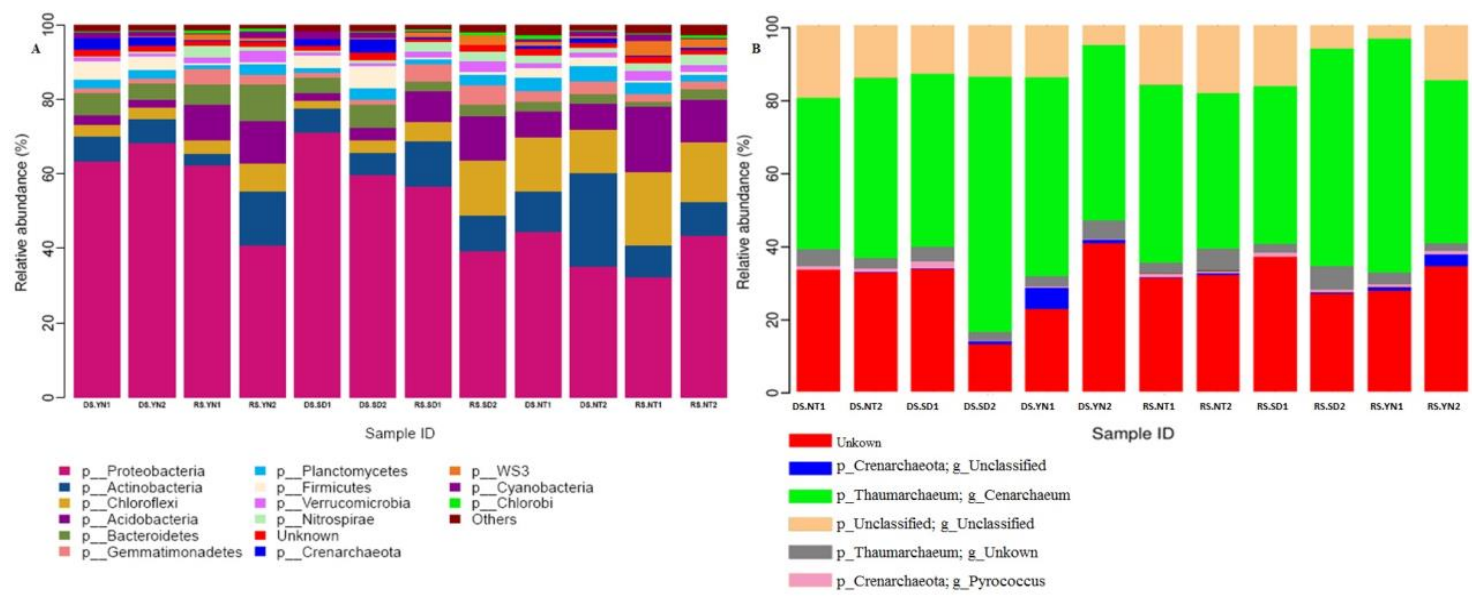

Figure 2. Relative abundance of bacterial phyla (A) and archaeal phyla (B) detected in soil samples. At different taxonomic levels, the classification of microbial community structure in soil samples to the currently known taxonomic unit is defined as "unknown"; the definition of "others" in which the proportion of microbial community structure is less than 1\%; although the microbial community information is aligned to sequences in the database, there is no definitive annotation for the data defined as "unclassified"

\section{Microbial community analysis of OTU distributions}

Based on the statistics of the total OTUs, Venn diagrams were created to display the unique and OTUs were shared among samples (Fig. 4). Under a similarity threshold of 0.97 , the numbers of overlapping bacterial and archaeal OTUs in the rainy season (2,634 and 828, respectively) were more than in the dry season $(1,187$ and 741 , respectively) in the three soil types. For archaea, the YN sampling area contained more OTUs (1329) than the NT (1020), and SD (935) areas. Each sample contained specific OTUs. For bacteria, the NT sampling area contained more OTUs $(2,547)$ than the SD $(1,391)$ and YN $(1,368)$ areas in the dry season, while the YN sampling area contained more OTUs $(4,127)$ than the SD $(3,344)$ and NT $(2,919)$ areas in the rainy season.

The authors also performed heatmap cluster analysis to discern the community compositional differences (Fig. 5). The overall taxonomic diversity of the bacterial community was significantly higher than that of the archaeal community at the genus level. Bacterial and archaeal taxonomic diversity was considerably lower in the dry season compared to the rainy season. The heatmap of bacterial communities showed that Proteobacteria, Actinobacteria and Bacteroidetes were most differentiated between seasons. Additionally, bacterial communities differed slightly in the soil samples from the SD and YN sampling areas in the dry season, but were more irregular in the rainy season. In contrast, Thaumarchaeota (Cenarchaeum) was the predominant phylum in the archaeal communities. The abundance of archaeal was independent of seasons and soil types.

\section{Microbial community beta-diversity analysis}

Based on Weighted_Unifrac distances, the work assessed the similarities and differences in the composition of the microbial communities with respect to seasonal and spatial variations (Fig. 6). The UPGMA analysis showed that the diversity and composition of the bacterial community were more impacted by seasonal changes than 
by soil types, the opposite of the low-abundance archaeal community. The archaeal community composition did not differ in soil samples from the SD and YN sampling areas, but dissimilar to the NT sampling area.

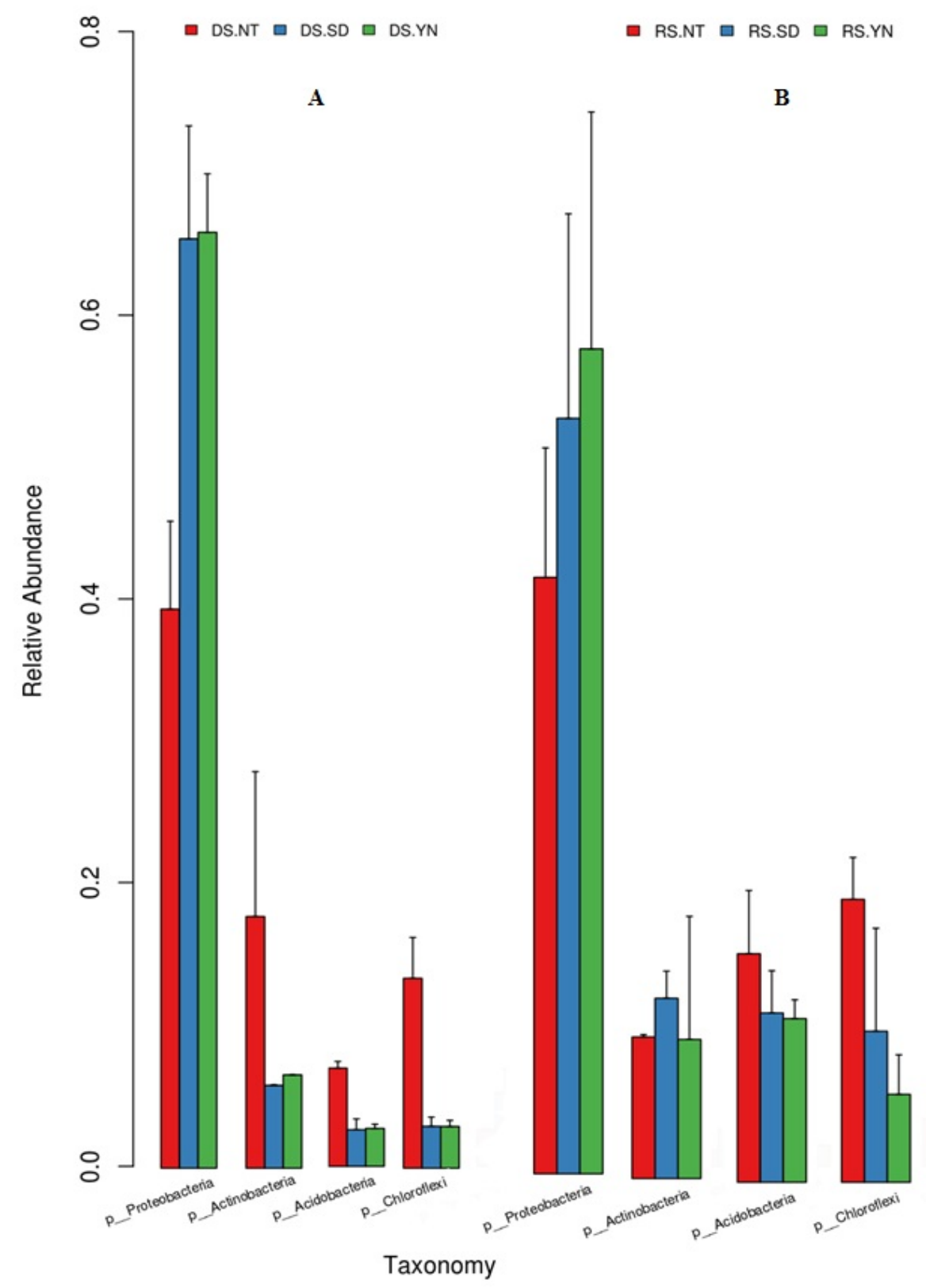

Figure 3. Comparisons of the bacteria four mainly phyla taxonomy abundance of soil samples $(Y N, S D$ and $N T)$ in dry and rainy seasons (DS and RS). The bacteria four mainly phyla taxonomy are Proteobacteria, Actinobacteria, Chloroflexi and Acidobacteria. A The soil samples are collected in dry season $(D S) . \boldsymbol{B}$ The soil samples are collected in rainy season (RS)

\section{Effects of environmental factors on microbial community diversity}

CCA was performed to identify the relationships between major environmental factors and microbial community diversity (Fig. 7). In the bacterial communities, the first two axes of the CCA plot explained 21.7 and $2.7 \%$ (in the dry season) and 77 and 
$26.92 \%$ (in the rainy season), respectively, of the total variation in the data. These results indicate that the bacterial community is closely correlated with $\mathrm{N}, \mathrm{TN}$ and SOM in the dry season. In the archaeal community, the first two axes of the CCA plot explained 34.38 and $27.05 \%$ (in the dry season) and 35.12 and $23.32 \%$ (in the rainy season), respectively, of the total variation in the data. However, no significant correlation of any of the factors was observed for the archaeal community.
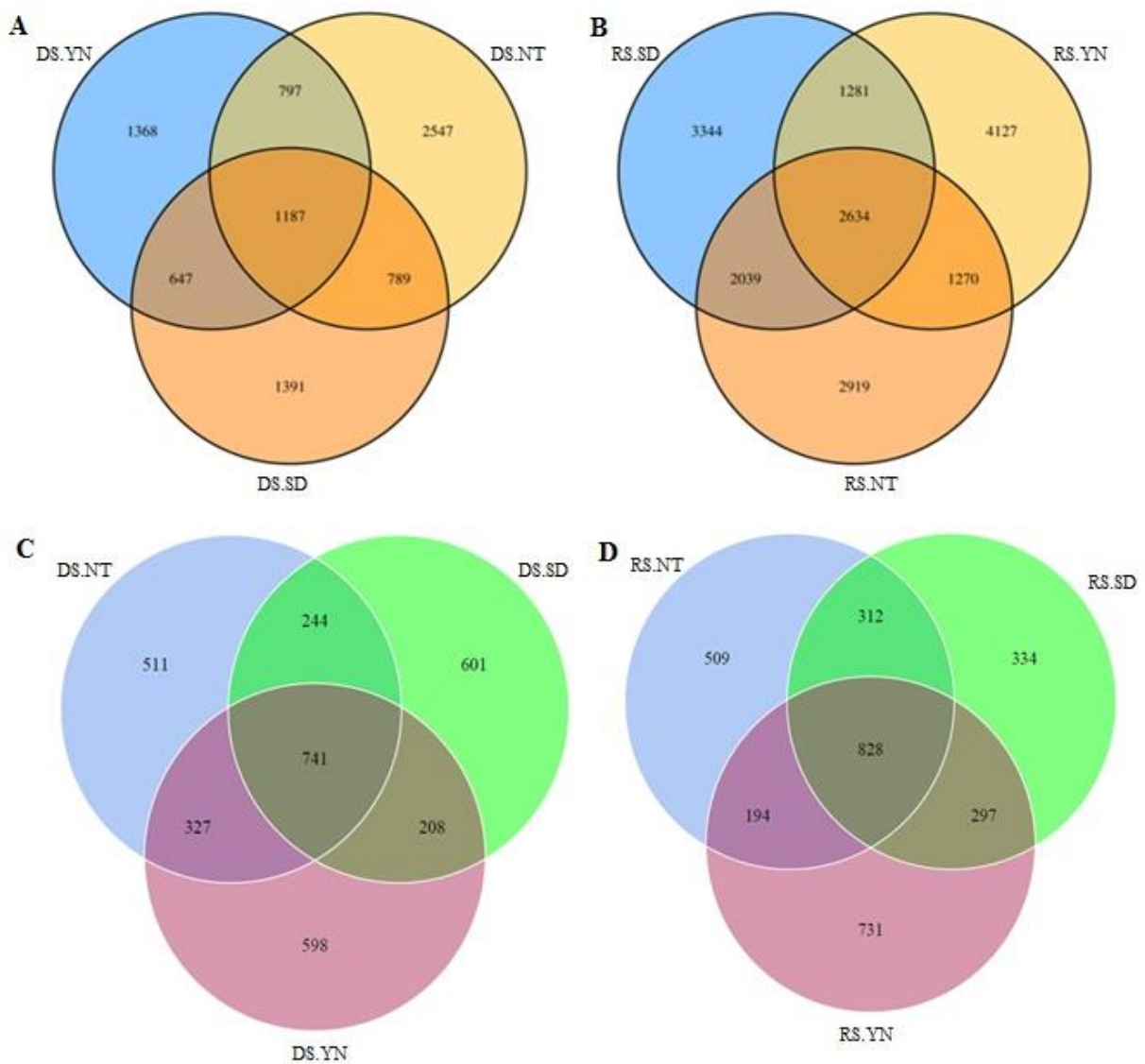

Figure 4. Venn diagram of microbial community of soil samples (YN, SD and NT) in dry and rainy seasons (DS and RS). The arrangement of the circles indicates the overlaps between the soil samples. A Venn diagram of bacterial community of soil samples in dry season (DS). $\boldsymbol{B}$ Venn diagram of bacterial community of soil samples in rainy season $(R S)$. C Venn diagram of archaeal community of soil samples in dry season $(D S)$. $\boldsymbol{D}$ Venn diagram of archaeal community of soil samples in rainy season $(R S)$

\section{Discussion}

Plant and animal diversity have been studied over the past decade in the Napahai plateau wetland. The research has been complemented with microbial diversity information through the measurement of the bacterial and archaeal community composition and diversity, allowing for a better assessment of the whole area's ecosystem.

The composition and diversity of the microbial community in the Napahai plateau wetland were investigated using high-throughput sequencing of 16S rRNA V4 genes. Proteobacteria, Actinobacteria, Chloroflexi and Acidobacteria were the top four most abundant bacterial phyla (Fig. 2A). Similar results have been obtained in different land- 
use types of soils in North and South America (Lauber et al., 2009), agricultural and forest soils in Salta and Jujuy (Montecchia et al., 2015) and soils from Antarctic and Arctic (Teixeira et al., 2010; Yergeau et al., 2010). However, compared with those derived from cucumber rhizosphere soils, Proteobacteria, Bacteroidetes and Actinobacteria in the work were the most abundant phyla, accounting for over $70 \%$ (Tian et al., 2013). Nevertheless, Proteobacteria is the most common phylum in soils worldwide, consistent with our results (Janssen, 2006; Spain et al., 2009). Actinobacteria is not only the dominant phylum in low temperature environments but also the dominant phylum in freshwater ecosystems (Zwart et al., 2002; Johnson et al., 2007). The Napahai plateau wetland has an average temperature of approximate $5{ }^{\circ} \mathrm{C}$ with a freshwater lake, and its ecological environment is in line with Actinobacteria growth. Thus, Actinobacteria was also the most abundant phylum in the region. In contrast, the archaeal community compositions, especially the dominant phylum Thaumarchaeota, were clearly different from many other ecosystems, which tend to be dominated by Crenarchaeota and Euryarchaeota. The difference may be due to special geological features (Porat et al., 2010; Wang et al., 2010). Thaumarchaeota was the most abundant phylum accounting for almost 50\%, which played an important role in the biochemical cycling of carbon, nitrogen and other elements (Cabello et al., 2004). In the Thaumarchaeota, most of the known sequences belong to the ammoxidation archaea, involved in the ammoxidation of soil and affect the nitrogen cycle. Therefore, it is speculated that archaea play a more important role than bacteria in the cycling of nitrogen in this area. How archaea and bacteria coordinate with each other to accomplish biogeochemical cycling in the wetland needs further study.

Moreover, greater biodiversity in soil can lead to a more stable system, providing the enhanced combinations of vital microbial functions and processes (Wagg et al., 2014; Regar et al., 2019). In the work, the difference in microbial community composition in different soil types revealed the feedback mechanism of ecological environmental degradation and microbial composition. Acidobacteria distribution in acidic environments is contaminated by heavy metal, belonging to non-original colonies (Diamond et al., 2019). Among the YN, SD and NT sampling areas, no significant differences in Acidobacteria abundance were observed (Fig. 3B). This may indicate that the whole area is affected by heavy metal pollution. In contrast, Proteobacteria and Chloroflexi showed differences $(p<0.1)$ in different soil types (Fig. 3B). One of the predominant classes of Proteobacteria was Gammaproteobacteria, which can degrade organic matter and were most abundant in the NT sampling area (Picazo et al., 2019). The authors also observed that the abundance of Chloroflexi was significantly higher in the NT sampling area, and these bacteria are known to live in environment with high organic matter content (Denef et al., 2015). These similar results could probably be due to the NT sampling area having more nitrogen oxides caused by serious disturbance of human activities. In the archaeal community, Methanofollis only present in the NT sampling area in the rainy season (Fig. 5B). Methanofollis lived in anaerobic environments such as oceans, lake sediments and the digestive tract of ruminants (Pazinato et al., 2010). In the NT sampling area, there are a large number of animals such as cattle and sheep grazing in the rainy season; therefore, Methanogenus has had an ecological balance with host ruminants for a long time. All the results illustrated that from the microbial perspective, the NT sampling area can be regarded as consisting of meadow soil formed due to disruption by human activities. Soil microbial composition is important for soil quality and health. 


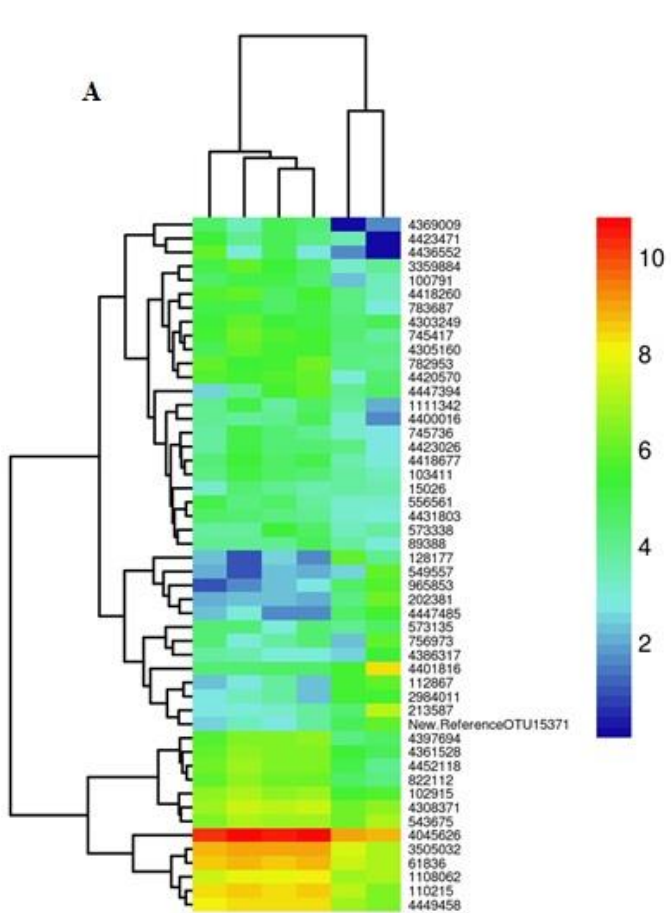

品品品品品品

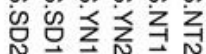

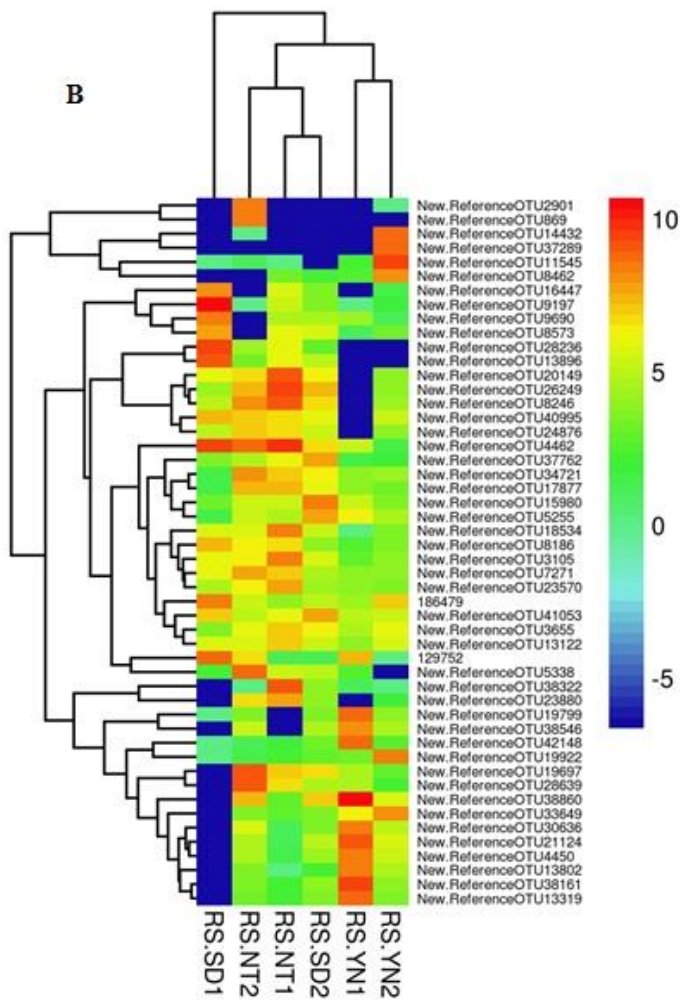

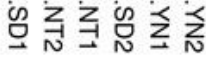

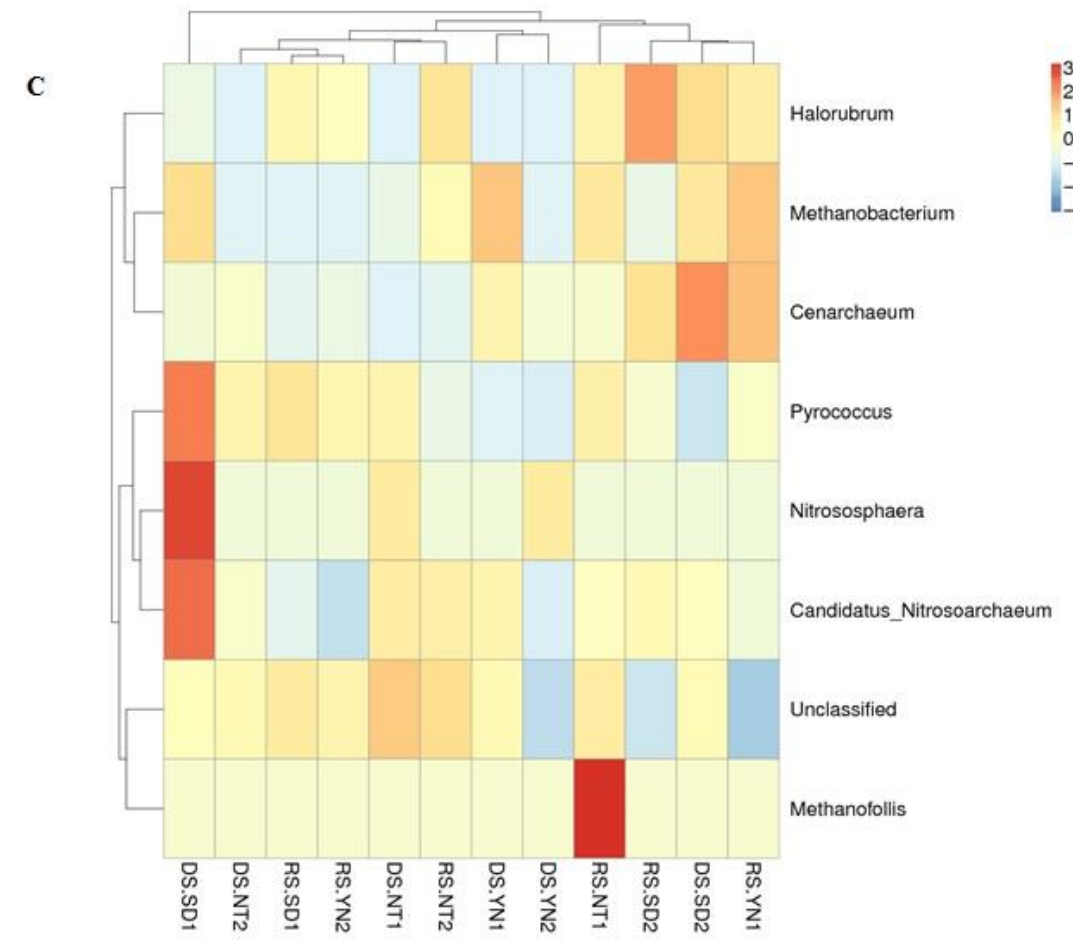

Figure 5. Heatmap cluster of microbial community of soil samples (YN, SD and NT) in dry and rainy seasons (DS and $R S$ ). A show the distribution of community OTUs classified at the genus level. Colors correspond to the relative abundance of each OTU within the community and cluster distance based on Bray-Curtis distances. Only the most abundant 50 bacterial genera based on statistical analysis are shown and the numbers in the plate to stand for in supplement material. A Heatmap cluster of bacterial community of soil samples in dry season (DS). $\boldsymbol{B}$ Heatmap cluster of bacterial community of soil samples in rainy season (RS). C Heatmap cluster of archaeal community of soil samples in dry and rainy seasons (DS and RS) 


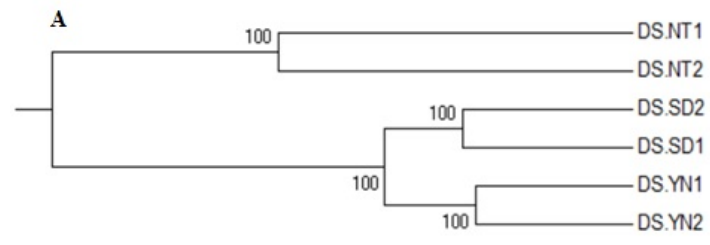

0.05
B

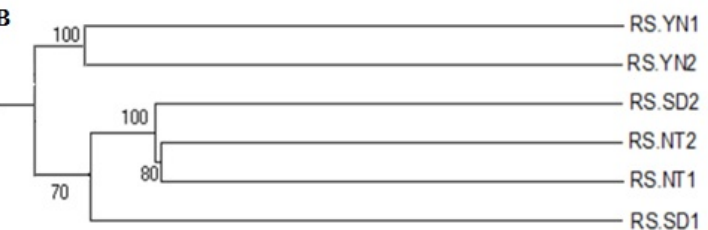

005

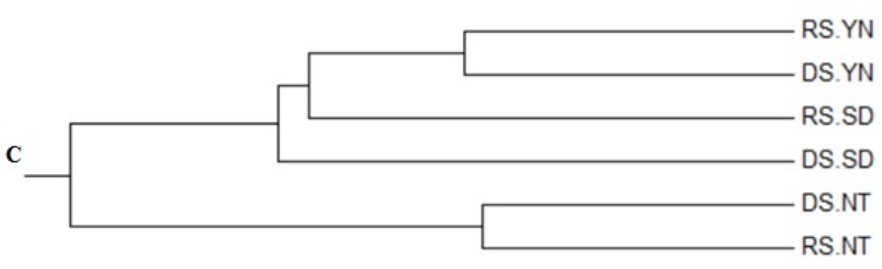

0.02

Figure 6. UPGMA of microbial community of soil samples (YN, SD and NT) in dry and rain seasons (DS and RS). A UPGMA of bacterial community of soil samples in dry season (DS). $\boldsymbol{B}$ UPGMA of bacterial community of soil samples in rainy season (RS). C UPGMA of archaeal community of soil samples in dry and rainy seasons (DS and RS)

In this work, the effects of seasons and soil types on soil microbial community characteristics were observed. Based on the analysis of OTU distributions (Venn diagrams and heatmap cluster) and beta-diversity analysis, the differences in two factors impact the microbial community (Figs. 4-6). Seasonal differences clearly impacted bacterial diversity, consistent with most of the literature reported (Bissett et al., 2007; Kara et al., 2013; Wilhelm et al., 2014). Unlike our result for bacterial diversity, archaeal diversity was affected by neither seasons nor soil types, especially in terms of abundance. Our finding is consistent with the numerous studies on the homogeneous spatial distributions of the archaeal community in various habitats, including field soil (Yuan et al., 2009) and sediment (Keuter et al., 2016); however, in ocean ecosystems, the diversity of archaea was influenced by spatial and temporal differences (Vik et al., 2017). This difference is probably due to unpredictable movements in ocean ecosystems. The soil ecosystem is relatively stable. However, one major drawback of our study was that the small number of sampling sites was in different soils samples, so the changes of microbial community diversity was relatively less convincing.

Our analysis of the relationship between major environmental factors and microbial community diversity showed that TN had the largest effect on bacterial community diversity, but the archaeal community was not significantly affected by environmental factors. This conclusion was also supported by our finding that archaeal diversity was not affected by seasons or soil types (Keuter and Rinkevich, 2016).

Finally, a large number of unclassified microorganisms were detected in the Napahai plateau wetland, with the abundance of unclassified bacteria and archaea at the phylum level being approximate 10 and 25\%, respectively. The unclassified bacteria consisted of the novel candidate divisions WPS-2, WS3, GN04, MVP-21 and MVS-104, whereas the archaeal candidate divisions included GA55, VAL11 and FRD15. Their ecological and biological interactions are unknown. Therefore, the isolation of soil microbes combined with traditional methods under laboratory conditions is ongoing, which will help us to identify critical resources in the Napahai plateau wetland. 
A
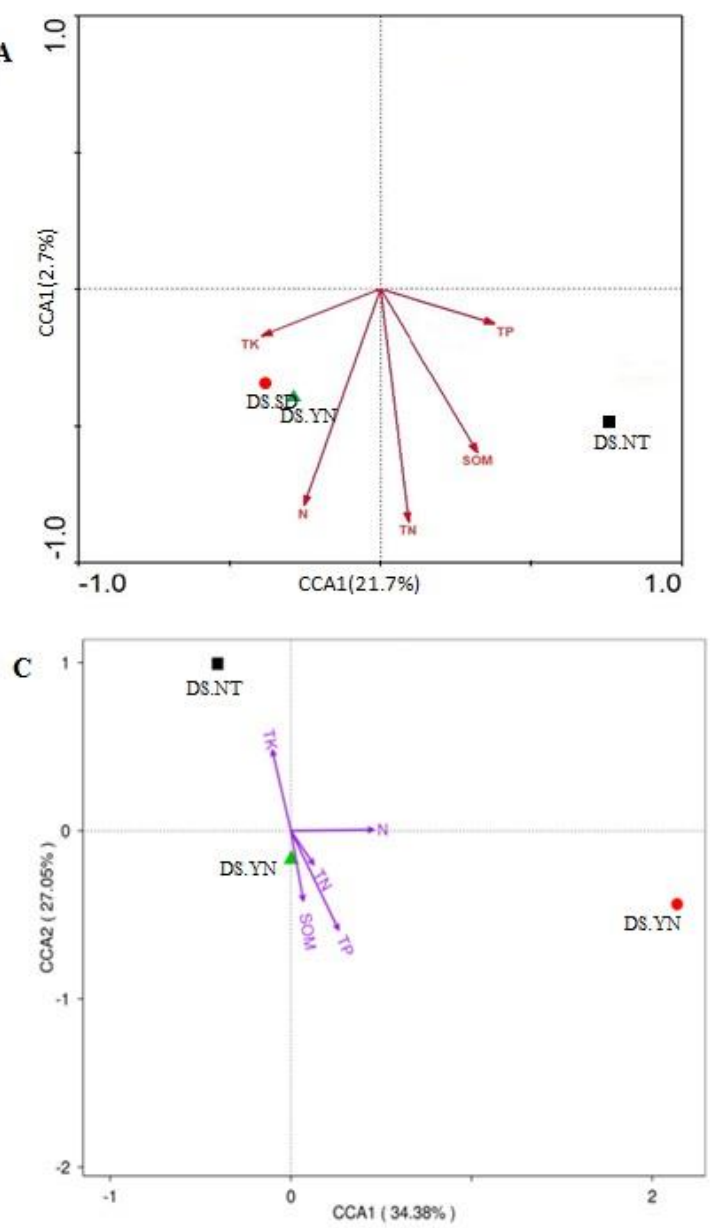
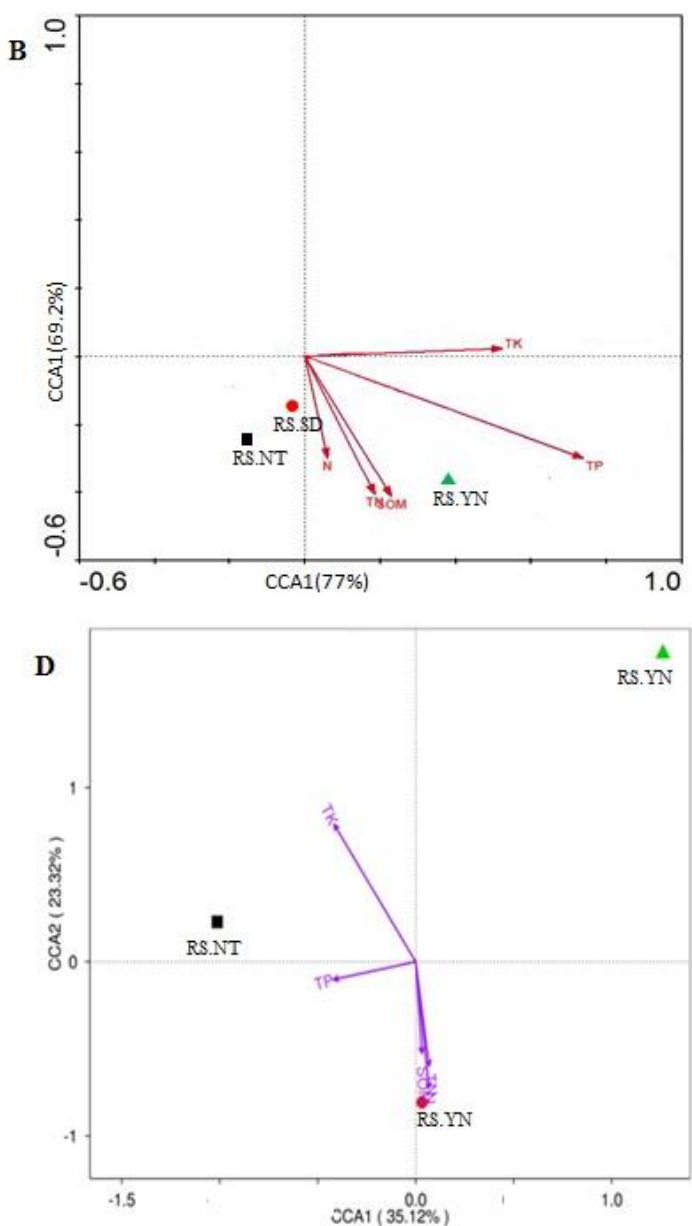

Figure 7. CCA plot of the relationship between the major environmental factors and microbial community composition of soil samples (YN, SD and NT) in dry and rain seasons (DS and RS).

Samples are indicated by dots and environmental factors indicated by color arrows. Arrow vector length represents the strength of the correlation with the axes. A CCA plot of the relationship between the major environmental factors and bacterial composition of soil samples in dry season $(D S) . \boldsymbol{B} C C A$ plot of the relationship between the major environmental factors and bacterial composition of soil samples in rainy season $(R S) . C C C A$ plot of the relationship between the major environmental factors and archaeal composition of soil samples in dry season $(D S)$. $\boldsymbol{D} C C A$ plot of the relationship between the major environmental factors and archaeal composition of soil samples in rainy season $(R S)$

\section{Conclusions}

The work examined the composition and diversity of the bacterial and archaeal communities with respect to spatial and temporal distribution and explored the relationships between environmental variables in the Napahai plateau wetland. For the bacterial community, Proteobacteria prevailed was the most abundant, followed by Actinobacteria, Chloroflexi and Acidobacteria. Meanwhile, they were more influenced by seasonal changes than by soil types and was closely correlated with N, TN and SOM in the dry season. For the archaea community, Thaumarchaeota dominated. Meanwhile, the archaeal community was neither associate with these factors nor significant correlation of physical and chemical factors. The microbial communities showed high and unique microbial diversity and composition, and influence with seasons and soil 
types, which lays a foundation for understanding the microbial and environmental response mechanism of the Napahai plateau wetland.

In the future, we will combine transcriptomics and proteomics to conduct research on microbial communities, and understand how the main bacteria or archaea conduct ecological functions in the particular ecological environment, and also for the discovery of unknown microorganism.

Acknowledgments. This research was funded by the Personnel Training Fund by the Kunming University of Science and Technology (KKSY201632059) and National Natural Science Foundation of China (No. 31700324).

\section{REFERENCES}

[1] Anderson, J. A., Hooper, M. J., Zak, J. C., Cox, S. B. (2009): Characterization of the structural and functional diversity of indigenous soil microbial communities in smelterimpacted and nonimpacted soils. - Environ Toxicol Chem 28(3): 534-541.

[2] Bissett, A., Burke, C., Cook, P. L., Bowman, J. P. (2007): Bacterial community shifts in organically perturbed sediments. - Environ Microbiol 9(1): 46-60.

[3] Cabello, P., Roldan, M. D., Moreno-Vivian, C. (2004): Nitrate reduction and the nitrogen cycle in archaea. - Microbiology 150(Pt 11): 3527-3546.

[4] Cao, H., Chen, R., Wang, L., Jiang, L., Yang, F., Zheng, S., Wang, G., Lin, X. (2016): Soil $\mathrm{pH}$, total phosphorus, climate and distance are the major factors influencing microbial activity at a regional spatial scale. - Sci Rep 6: 25815.

[5] Caporaso, J. G., Kuczynski, J., Stombaugh, J., Bittinger, K., Bushman, F. D., Costello, E. K., Fierer, N., Pena, A. G., Goodrich, J. K., Gordon, J. I., Huttley, G. A., Kelley, S. T., Knights, D., Koenig, J. E., Ley, R. E., Lozupone, C. A., McDonald, D., Muegge, B. D., Pirrung, M., Reeder, J., Sevinsky, J. R., Turnbaugh, P. J., Walters, W. A., Widmann, J., Yatsunenko, T., Zaneveld, J., Knight, R. (2010): QIIME allows analysis of highthroughput community sequencing data. - Nat Methods 7(5): 335-336.

[6] Dai, Y., Yan, Z., Jia, L., Zhang, S., Gao, L., Wei, X., Mei, Z., Liu, X. (2016): The composition, localization and function of low-temperature-adapted microbial communities involved in methanogenic degradations of cellulose and chitin from Qinghai-Tibetan Plateau wetland soils. - J Appl Microbiol 121(1): 163-176.

[7] Danilova, O. V., Belova, S. E., Gagarinova, I. V., Dedysh, S. N. (2016): Microbial community composition and methanotroph diversity of a subarctic wetland in Russia. Mikrobiologiia 85(5): 545-554.

[8] Denef, V. J., Mueller, R. S., Chiang, E., Liebig, J. R., Vanderploeg, H. A. (2015): Chloroflexi CL500-11 populations that predominate deep-lake hypolimnion bacterioplankton rely on nitrogen-rich dissolved organic matter metabolism and $\mathrm{C} 1$ compound oxidation. - Appl Environ Microbiol 82(5): 1423-1432.

[9] Diamond, S., Andeer, P. F., Li, Z., Crits-Christoph, A., Burstein, D., Anantharaman, K., Lane, K. R., Thomas, B. C., Pan, C., Northen, T. R., Banfield, J. F. (2019): Mediterranean grassland soil C-N compound turnover is dependent on rainfall and depth, and is mediated by genomically divergent microorganisms. - Nat Microbiol 4: pages 1356-1367.

[10] Edgar, R. C. (2013): UPARSE: highly accurate OTU sequences from microbial amplicon reads. - Nat Methods 10(10): 996-998.

[11] Janssen, P. H. (2006): Identifying the dominant soil bacterial taxa in libraries of $16 \mathrm{~S}$ rRNA and 16S rRNA genes. - Appl Environ Microbiol 72(3): 1719-1728.

[12] Johnson, S. S., Hebsgaard, M. B., Christensen, T. R., Mastepanov, M., Nielsen, R., Munch, K., Brand, T., Gilbert, M. T. P., Zuber, M. T., Bunce, M. (2007): Ancient 
bacteria show evidence of DNA repair. - Proceedings of the National Academy of Sciences of the United States of America 104(36): 14401-14405.

[13] Kara, E. L., Hanson, P. C., Hu, Y. H., Winslow, L. McMahon, K. D. (2013): A decade of seasonal dynamics and co-occurrences within freshwater bacterioplankton communities from eutrophic Lake Mendota, WI. - ISME J 7(3): 680-684.

[14] Keuter, S., Rinkevich, B. (2016): Spatial homogeneity of bacterial and archaeal communities in the deep eastern Mediterranean Sea surface sediments. - Int Microbiol 19(2): 109-119.

[15] Lauber, C. L., Hamady, M., Knight, R., Fierer, N. (2009): Pyrosequencing-based assessment of soil $\mathrm{pH}$ as a predictor of soil bacterial community structure at the continental scale. - Appl Environ Microbiol 75(15): 5111-5120.

[16] Legendre, P., Gallagher, E. D. (2001): Ecologically meaningful transformations for ordination of species data. - Oecologia 129(2): 271-280.

[17] Montecchia, M. S., Tosi, M., Soria, M. A., Vogrig, J. A., Sydorenko, O., Correa, O. S. (2015): Pyrosequencing reveals changes in soil bacterial communities after conversion of Yungas forests to agriculture. - PLoS One 10(3): e0119426.

[18] Oksanen, J., Blanchet, F. G., Kindt, R., Legendre, P., O’Hara, R. B., Simpson, G. L., Solymos, P., Stevens, M. H., Wagner, H. (2013): Multivariate Analysis of Ecological Communities in R: package "vegan". - R Package Version 1.

[19] Pazinato, J. M., Paulo, E. N., Mendes, L. W., Vazoller, R. F., Tsai, S. M. (2010): Molecular characterization of the archaeal community in an Amazonian wetland soil and culture-dependent isolation of methanogenic archaea. - Diversity 2(7): 1026-1047.

[20] Peiffer, J. A., Spor, A., Koren, O., Jin, Z., Tringe, S. G., Dangl, J. L., Buckler, E. S., Ley, R. E. (2013): Diversity and heritability of the maize rhizosphere microbiome under field conditions. - Proc Natl Acad Sci USA 110(16): 6548-6553.

[21] Picazo, A., Rochera, C., Villaescusa, J. A., Miralles-Lorenzo, J., Velazquez, D., Quesada, A., Camacho, A. (2019): Bacterioplankton Community Composition Along Environmental Gradients in Lakes From Byers Peninsula (Maritime Antarctica) as Determined by Next-Generation Sequencing. - Front Microbiol 10: 908.

[22] Porat, I., Vishnivetskaya, T. A., Mosher, J. J., Brandt, C. C., Yang, Z. K., Brooks, S. C., Liang, L., Drake, M. M., Podar, M., Brown, S. D., Palumbo, A. V. (2010): Characterization of archaeal community in contaminated and uncontaminated surface stream sediments. - Microb Ecol 60(4): 784-795.

[23] Regar, R. K., Gaur, V. K., Bajaj, A., Tambat, S., Manickam, N. (2019): Comparative microbiome analysis of two different long-term pesticide contaminated soils revealed the anthropogenic influence on functional potential of microbial communities. - Sci Total Environ 681: 413-423.

[24] Savio, D., Sinclair, L., Ijaz, U. Z., Parajka, J., Reischer, G. H., Stadler, P., Blaschke, A. P., Bloschl, G., Mach, R. L., Kirschner, A. K., Farnleitner, A. H., Eiler, A. (2015): Bacterial diversity along a $2600 \mathrm{~km}$ river continuum. - Environ Microbiol 17(12): 49945007.

[25] Schloss, P. D., Westcott, S. L., Ryabin, T., Hall, J. R., Hartmann, M., Hollister, E. B., Lesniewski, R. A., Oakley, B. B., Parks, D. H., Robinson, C. J., Sahl, J. W., Stres, B., Thallinger, G. G., Van Horn, D. J., Weber, C. F. (2009): Introducing mothur: opensource, platform-independent, community-supported software for describing and comparing microbial communities. - Appl Environ Microbiol 75(23): 7537-7541.

[26] Shehab, N., Li, D., Amy, G. L., Logan, B. E., Saikaly, P. E. (2013): Characterization of bacterial and archaeal communities in air-cathode microbial fuel cells, open circuit and sealed-off reactors. - Appl Microbiol Biotechnol 97(22): 9885-9895.

[27] Spain, A. M., Krumholz, L. R., Elshahed, M. S. (2009): Abundance, composition, diversity and novelty of soil Proteobacteria. - ISME J 3(8): 992-1000.

[28] Su, J. Q., Ding, L. J., Xue, K., Yao, H. Y., Quensen, J., Bai, S. J., Wei, W. X., Wu, J. S., Zhou, J., Tiedje, J. M., Zhu, Y. G. (2015): Long-term balanced fertilization increases the 
soil microbial functional diversity in a phosphorus-limited paddy soil. - Mol Ecol 24(1): 136-150.

[29] Teixeira, L. C., Peixoto, R. S., Cury, J. C., Sul, W. J., Pellizari, V. H., Tiedje, J., Rosado, A. S. (2010): Bacterial diversity in rhizosphere soil from Antarctic vascular plants of Admiralty Bay, maritime Antarctica. - ISME J 4(8): 989-1001.

[30] Tian, Y., Gao, L. (2014): Bacterial diversity in the rhizosphere of cucumbers grown in soils covering a wide range of cucumber cropping histories and environmental conditions. - Microb Ecol 68(4): 794-806.

[31] Tian, Y., Zhang, X., Wang, J., Gao, L. (2013): Soil microbial communities associated with the rhizosphere of cucumber under different summer cover crops and residue management: a 4-year field experiment. - Scientia Horticulturae 150(2): 100-109.

[32] Vik, D. R., Roux, S., Brum, J. R., Bolduc, B., Emerson, J. B., Padilla, C. C., Stewart, F. J., Sullivan, M. B. (2017): Putative archaeal viruses from the mesopelagic ocean. - PeerJ 5: e3428.

[33] Wagg, C., Bender, S. F., Widmer, F. van der Heijden, M. G. (2014): Soil biodiversity and soil community composition determine ecosystem multifunctionality. - Proc Natl Acad Sci USA 111(14): 5266-5270.

[34] Wang, P., Li, T., Hu, A., Wei, Y., Guo, W., Jiao, N., Zhang, C. (2010): Community structure of archaea from deep-sea sediments of the South China Sea. - Microb Ecol 60(4): 796-806.

[35] Widder, S., Besemer, K., Singer, G. A., Ceola, S., Bertuzzo, E., Quince, C., Sloan, W. T., Rinaldo, A., Battin, T. J. (2014): Fluvial network organization imprints on microbial cooccurrence networks. - Proc Natl Acad Sci USA 111(35): 12799-12804.

[36] Wilhelm, S. W., LeCleir, G. R., Bullerjahn, G. S., McKay, R. M., Saxton, M. A., Twiss, M. R., Bourbonniere, R. A. (2014): Seasonal changes in microbial community structure and activity imply winter production is linked to summer hypoxia in a large lake. - FEMS Microbiol Ecol 87(2): 475-485.

[37] Xiang, Y., Wang, S., Li, J., Wei, Y., Zhang, Q., Lin, L., Ji, X. (2018): Isolation and characterization of two lytic cold-active bacteriophages infecting Pseudomonas fluorescens from the Napahai plateau wetland. - Can J Microbiol 64(3): 183-190.

[38] Yergeau, E., Hogues, H., Whyte, L. G., Greer, C. W. (2010): The functional potential of high Arctic permafrost revealed by metagenomic sequencing, qPCR and microarray analyses. - ISME J 4(9): 1206-1214.

[39] Yuan, Y., Conrad, R., Lu, Y. (2009): Responses of methanogenic archaeal community to oxygen exposure in rice field soil. - Environ Microbiol Rep 1(5): 347-354.

[40] Zhao, J., Ni, T., Li, Y., Xiong, W., Ran, W., Shen, B., Shen, Q., Zhang, R. (2014): Responses of bacterial communities in arable soils in a rice-wheat cropping system to different fertilizer regimes and sampling times. - PLoS One 9(1): e85301.

[41] Zhou, Z., Meng, H., Liu, Y., Gu, J. D., Li, M. (2017): Stratified bacterial and archaeal community in mangrove and intertidal wetland mudflats revealed by high throughput $16 \mathrm{~S}$ rRNA gene sequencing. - Front Microbiol 8: 2148.

[42] Zwart, G., Crump, B. C., Agterveld, M., Hagen, F., Han, S. K. (2002): Typical freshwater bacteria: an analysis of available $16 \mathrm{~S}$ rRNA gene sequences from plankton of lakes and rivers. - Aquatic Microbial Ecology 28(2): 141-155. 